\title{
Physiological and behavioral responses to routine procedures in captive common marmosets (Callithrix jacchus)
}

\author{
Ana Cecília de Menezes Galvão ${ }^{1} \cdot$ Renata Gonçalves Ferreira $^{1}$. \\ Maria Bernardete Cordeiro de Sousa ${ }^{1} \cdot$ Nicole Leite Galvão-Coelho $^{1}$
}

Received: 17 June 2015/Accepted: 22 February 2016/Published online: 5 March 2016

(C) Japan Monkey Centre and Springer Japan 2016

\begin{abstract}
The effect of routine captive procedures on the welfare of species used as experimental models in biomedical research is of great interest, since stress may alter the generalization and interpretation of results. This study investigated behavioral and endocrine (fecal cortisol) reactivity patterns in common marmoset (Callithrix jacchus) adult males $(N=10)$ and females $(N=9)$ subjected to three types of routine procedures in captivity: (1) moving to a same-sized cage (P1), to a smaller cage (P2), and (2) first-time pair formation (P3). Sexually dimorphic cortisol responses were detected in animals submitted to a physical environmental stressor (cage change). Females showed an increased response throughout $\mathrm{P} 1$, in relation to baseline (BP) cortisol, and a trend during P2. Males increased cortisol only during P2. On the other hand, males and females showed a similar endocrine response when management involved social challenge (pair formation), with both sexes increasing cortisol levels, but females exhibited a more intense and longer-lasting cortisol increase. Males and females exhibited similar behavioral responses to cage change, except for autogrooming, with males decreasing this behavior in P1. Only females demonstrated a significantly higher increase in piloerection frequency than that of males during the pair formation phase. These endocrine and behavioral changes must be taken into account when interpreting research data that involve these types of procedures. Further studies on the impacts of routine colony management are required to
\end{abstract}

Nicole Leite Galvão-Coelho

galvaonic@hotmail.com

1 Department of Physiology, Psychobiology Graduation Program, Federal University of Rio Grande do Norte, Caixa Postal, 1511, Natal, RN 59078-970, Brazil devise and include protocols in official husbandry guidelines.

Keywords Behavior - Colony management $\cdot$ Fecal cortisol $\cdot$ Non-human primates $\cdot$ Stress

\section{Introduction}

Conditions of captivity are typically characterized by social isolation or high population density, low predatory pressure, and easy access to food (Newberry 1993). Furthermore, experimental protocols generally dictate that nonhuman primates used in biomedical research be confined in isolation in small cages (Baker 2007; Baker et al. 2007). These conditions are usually foreign to the natural environment of model species and can induce changes in organic and cognitive functions as well as behavioral changes (Boere 2001). Although the possible bias caused by unrealistic captivity conditions in investigational studies has been largely acknowledged, the impact of routine husbandry procedures on experimental studies is not well known (Ruby and Buchanan-Smith 2015). Balcombe et al. (2004) found that animals exhibited signs of distress after routine husbandry procedures, such as spatial constraints to the environment during cage cleaning or handling for blood sampling and oral gastric lavage. Certain signs of distress such as plasma corticosteroid concentrations, heart rate, blood pressure, and specific behaviors increased by $20-100 \%$ compared to baseline and lasted for $30 \mathrm{~min}$ or more. The authors discuss these findings as to whether such changes in baseline values can bias biomedical research outcomes.

Stress, a bodily arousal mechanism activated in response to internal and/or external challenges, involves primarily 
the stimulation of neuroendocrine and autonomic pathways. These mechanisms sustain physiological and behavioral responses in order to overcome the challenges presented, and restore homeostasis (Charmandari et al. 2005). When changes triggered by the stress response are acutely activated, they may be beneficial to the individual. However, when these changes are maintained chronically, they may result in biological, psychological, and behavioral imbalance, as well as give rise to the emergence of physical and mental illness, compromising animal wellbeing (McEwen 2000).

A less invasive indicator of an animal's clinical condition involves examining the behavioral repertoire of a species (Dawkins 2004). Abnormal ethological patterns, in addition to the expression of stereotypic behaviors and/or self-mutilation, may suggest poor welfare and the presence of cognitive disorders (Young 2003). Examining hormonal profiles generally corroborates behavioral indicators. For example, it has been shown that changes in fecal cortisol associated with social changes can be recorded (Sousa and Ziegler 1998). Hormonal profiles also contribute to welfare assessment since the use of non-invasive techniques to measure fecal steroid hormones (cortisol, progesterone, and androgens) makes it possible to evaluate both reproductive status and stress levels (Sousa and Ziegler 1998).

Callithrix jacchus, a small Neotropical primate endemic to the Brazilian Atlantic Rainforest (Stevenson and Rylands 1988), has been used for decades as a model species in a number of biomedical fields (Mansfield 2003; Prasad et al. 2007; Kohu et al. 2008). The species is commonly used as a test animal due to its good adaptation to captivity, high fertility rate, and relatively small size (Dixson and Lunn 1987; Lacreuse et al. 2014). This species has a well-defined ethogram (Stevenson and Poole 1976), and non-invasive techniques to measure fecal steroid hormones (cortisol, progesterone, and androgens) are well established, promoting the use of this species as an experimental model ('t Hart et al. 2012; Kishi et al. 2014; Phillips et al. 2015).

Considering the frequent use of $C$. jacchus as an animal model, the current study aims to investigate their reactivity to routine handling in captivity such as being moved to similar or smaller-size cages, and first-time pair formation. Based on individual reactivity and differences in behavior and cortisol profiles between males and females faced with stressful situations, as demonstrated in the literature, we tested three hypotheses: First, moving common marmosets to a spatially restricted cage will be a more severe stressor than moving to a new environment with no spatial restriction. Second, males and females will show different patterns (intensity and temporality) of endocrine and behavioral response to cage changes. Third, males and females will exhibit an acute dimorphic stress response to first-time pair formation.

\section{Methods}

\section{Animal maintenance}

Ten adult common marmoset (Callithrix jacchus) males and nine females, without reproductive experience and unfamiliar with each other, were used in the current study. All animals were held in captivity at the Laboratory for Advanced Primates Study of the Federal University of Rio Grande do Norte (UFRN), northeastern Brazil. The marmosets occupied outdoor masonry cages measuring $2.0 \times 2.0 \times 1.0 \mathrm{~m}$, maintained under natural lighting, humidity, and temperature conditions. The cages had a wire mesh roof and a one-way mirror on the front masonry wall through which behavioral observations could be made. The back wall had a stainless steel door where the drinking fountain and a feed plate were located. At the beginning of the study, all animals were socially isolated, with no visual contact, but were allowed acoustic and olfactory contact during the previous 3 months or longer (depending on the beginning of the study). Before the animals were moved, all cages were cleaned using $90 \%$ ethanol. Food, consisting of a nutritious mixture (oat, soy, milk, egg, and bread) and fruits, was provided twice a day. Water was available ad libitum.

The animals were housed according to IBAMA (Brazilian Institute of Environment and Renewable Natural Resources) guidelines (Normative Instruction no. 169 of February 20, 2008), and the care standards for animals established by CONCEA-National Council for Animal Experimentation Control, Law No. 11.794 (October 8, 2008). In addition, the laboratory complies with international standards for ex-situ maintenance of animals defined by the Animal Behavior Society and the International Primatological Society. The animals were assessed by a veterinarian throughout the study and experimental procedures were approved by the Animal Research Ethics Committee (UFRN protocol No. 018/2012).

All animals from both experiments were socially isolated before the study for an average of $32.7 \pm 4.3$ and $24.0 \pm 5.7$ months for experiment I and experiment II, respectively, either because they had participated in previous scientific studies that required this condition, or were wild animals introduced into captivity.

\section{Study design}

Experiment I: moving between cages (females: $n=5$; males: $n=6$ )

Baseline phase (BP) During this 27-day period, the animals were isolated in masonry cages measuring $2.0 \times 2.0 \times 1.0 \mathrm{~m}$, and both behavioral observations and 
fecal samplings were performed on alternate days (totaling 14 days of data collection).

Moving to same-size cages (P1) After the BP, animals were moved to a masonry cage of the same size $(2.0 \times 2.0 \times 1.0 \mathrm{~m})$ and complexity, where they remained for 7 days. Behavioral data and fecal samples were collected daily.

Moving to small-size cages (P2) Following P1, animals were moved to a smaller stainless steel cage measuring $42 \times 68 \times 87 \mathrm{~cm}$. This small cage was placed inside the previous cage (P1) to avoid the influence of variables other than space restriction. Thus, in this phase the animal remained under the same visual, acoustic and/or olfactory environment. This phase also lasted 7 days and behavioral and fecal samplings were conducted daily.

\section{Experiment II: first-time pair formation (male/female pairs $=4$ )}

Baseline phase 2 (BP2) All animals were isolated in masonry cages, measuring $2.0 \times 2.0 \times 1.0 \mathrm{~m}$, for 30 days, during which both behavioral observations and fecal samplings were performed on alternate days.

First-time pair formation phase (P3) At the end of BP2, the animals were paired in a new same-size cage. This phase lasted 7 days and behavioral data and fecal samples were taken daily.

\section{Behavioral data collection}

Continuous focal sampling method was used to evaluate the effect of changes in physical environment on behavior, with the frequency or duration of selected behaviors recorded continuously for each animal over a 30-min period (Altmann 1974). Behavioral data were always collected between 06:30 and 08:30 a.m. to avoid the influence of circadian variation (Erkert 1989). The behaviors were described according to the ethogram compiled by Stevenson and Poole (1976). Individual behaviors, including autogrooming (duration), scent marking (frequency), and piloerection (frequency), were analyzed. Two observers participated in data collection throughout the study, resulting in $90 \%$ interobserver accuracy.

\section{Fecal collection and cortisol assay}

Feces were collected every morning between 6:30 and 8:30 a.m. to avoid circadian variation in cortisol. Prior to fecal collection, the cages were cleaned to facilitate sample identification. The observer entered the cage shortly after animal defecation and collected the sample with a wooden stick. Samples were identified and stored at approximately $-4{ }^{\circ} \mathrm{C}$, and then processed for cortisol extraction and quantification at the Hormonal Measurements Laboratory, Department of Physiology-UFRN.

Briefly, the fecal extraction protocol consisted of weighing $0.1 \mathrm{~g}$ of feces per sample in a labeled $15-\mathrm{mL}$ plastic tube. The first step in hydrolysis is extraction, which consists of separating the steroids (cortisol) in the free form from the water soluble substances. This is followed by solvolysis, which separates steroid conjugates. Finally, the cortisol extract is resuspended in alcohol, and samples are stored until the enzyme-immunoassay is performed according to the protocol proposed by Raminelli et al (2001). The coefficients of variation were 11.58 and $7.9 \%$ for intra- and interassays, respectively.

\section{Statistical analyses}

The Statistica 7 software package was used. In order to match the other study phases for hormonal and behavioral baseline phase data (BP and BP2), an average was calculated every 2 days, totaling seven sets of data in each phase, for each test. The general linear model test (GLM) and Fisher's post-hoc test were conducted to analyze cortisol level variations. The "sex" variable was used as an independent factor, while cortisol levels as well as frequency and durations of behaviors across the phases were the dependent variables. One outlier value of four standard deviations above the mean, recorded for a single female, was excluded from the baseline phase of experiment II (BP2). Moreover, a qualitative statistical analyses of the progression of cortisol response in P2 and P3 was made.

The nonparametric Mann-Whitney test was used to investigate the influence of "sex" on behavioral data. Friedman's analysis of variance (ANOVA) test was applied to examine behavioral changes across the phases as a result of changing cage sizes (experiment I: BP, P1, P2) and the Wilcoxon test was performed for data related to first-time pair formation (experiment II: BP, P2, P3). Spearman's correlation test was used to analyze possible correlations between behavior and cortisol across study phases and to analyze the influence of environmental variables (temperature, humidity, and rainfall) on cortisol levels.

A statistically significant result was considered to be $p \leq$ 0.05 . A statistically result was considered trend when 0.05 $<p \leq 0.07$ in all the tests.

The study was conducted in 2010 and 2011, but each animal was tested for only 35-41 days, depending on whether they were engaged in experiment I or II. To avoid possible climatic confounding factors in the behavioral and/or cortisol profiles, Spearman's correlation tests were conducted between climatic and biological variables and 
data. Irrespective of sex, a weak negative correlation was found between cortisol and average daily temperature (Spearman's correlation, $p=0.0001, r \mathrm{~s}=-0.29$ ) in all the 2010 values, as well as tendency for a weak positive correlation between cortisol and humidity (Spearman's correlation, $\mathrm{p}=0.056, r \mathrm{~s}=0.12$ ). No correlations were found between cortisol and daily rainfall (Spearman's correlation, $p=0.1, r \mathrm{~s}=0.07$ ) in 2010. In 2011, no correlation was detected between cortisol and average daily temperature (Spearman's correlation, $p=0.506$, $r \mathrm{~s}=-0.07$ ), cortisol and average daily humidity (Spearman's correlation, $p=0.555, r \mathrm{~s}=-0.06$ ) or cortisol and average daily rainfall (Spearman's correlation, $p=0.661$, $r \mathrm{~s}=-0.04)$. Considering the significant correlations and the fact that the animals were used only for 35-41 successive days, it was assumed that neither cortisol nor behavioral changes were significantly affected by tested climatic variables.

\section{Results}

\section{Experiment I: moving between cages}

\section{Cortisol}

Females showed higher total cortisol levels $(\mu=42.59 \pm$ $2.87 \mathrm{ng} / \mathrm{g})$ than males $(\mu=13.09 \pm 2.52 \mathrm{ng} / \mathrm{g})(\mathrm{GLM}$; sex: $\left.F=4.92, d f=1, p=0.02, \eta_{p}^{2}=0.024\right)$ after moving to a new cage. Males and females exhibited a statistical difference in response to moving to new cages in terms of cortisol levels (GLM; sex*phases: $F=4.24, d f=2$, $p=0.01, \quad \eta_{p}^{2}=0.041$ ). Males obtained a significant increase in cortisol levels during $\mathrm{P} 2$ when compared to $\mathrm{BP}$ and P1 (Fisher's post-hoc: P1*BP: $p=0.9 ; \quad \mathrm{P} 2 * \mathrm{BP}$ : $p=0.02 ; \mathrm{P} 2 * \mathrm{P} 1 ; p=0.02)$. Females showed a significant rise in cortisol levels in P1 with respect to BP (Fisher's post-hoc: $\mathrm{P} 1 * \mathrm{BP}: p=0.001)$ and a statistical trend toward an increase in $\mathrm{P} 2$ when compared to BP (Fisher's post-hoc: P2*BP: $p=0.07$ ). No differences were observed between $\mathrm{P} 1$ and P2 for females (Fisher's post-hoc: P2*P1: $p=0.15$ ) (Fig. 1a), although the observed values reveal that the cortisol profile in P2 was lower than that of P1 (Fig. 1b). Females also showed higher cortisol levels than males in P1 (Fisher's post-hoc: BP: $p=0.84$; P1: $p=0.0004 ; \mathrm{P} 2: p=0.96$ ). A qualitative analysis of the progression of cortisol response shows that males exhibited a sharp increase that returned to baseline cortisol levels on the day after they moved to a small cage (P2), according to a quadratic distribution $(F=40.321, \quad p=0.002$, $\beta=-3.906$ ), as illustrated in Fig. 1b. On the other hand, females displayed a more variable and longer-lasting cortisol response after changing cages (P2), which could not be well fit to any distribution $(\beta=-0.255$, but curve estimation tests were all non-significant), as depicted in Fig. 1b.

\section{Behaviors}

Scent marking No sex differences were detected in total scent marking episodes (Mann-Whitney, $U=6527$, $p=0.861$ ). However, males showed a significant interphase difference in scent marking frequency (Friedman ANOVA: $\chi^{2}=31.299, d f=2, p=0.001$ ), reducing this behavior during $\mathrm{P} 2$ in relation to $\mathrm{BP}$ and $\mathrm{P} 1$ (Wilcoxon: $\mathrm{B} * \mathrm{P} 2 ; \quad z=-4.3221, \quad p=0.001 ; \quad \mathrm{P} 1 * \mathrm{P} 2 ; z=-4.406$, $p=0.001$ ), but no variation was observed during $\mathrm{P} 1$ in relation to $\mathrm{BP}$ (Wilcoxon: $\mathrm{BP} * \mathrm{P} 1 ; z=-0.842, p=0.399$ ) (Fig. 2a). Females also showed a significant difference in scent marking frequency across the phases (Friedman ANOVA: $\chi^{2}=18.84, d f=2, p=0.001$ ), decreasing this behavior during $\mathrm{P} 2$ compared to $\mathrm{BP}$ and $\mathrm{P} 1$ (Wilcoxon: $\mathrm{BP} * \mathrm{P} 2 ; \quad z=-3.528, \quad p=0.001 ; \mathrm{P} 1 * \mathrm{P} 2 ; z=-3.846$, $p=0.001$ ) but not between $\mathrm{P} 1$ and $\mathrm{BP}$ (Wilcoxon: $\mathrm{BP} * \mathrm{P} 1$; $z=-0.569, p=0.570$ ) (Fig. 2a).

Individual piloerection No sex differences were detected between total individual piloerection and total event frequency (Mann-Whitney, $U=6089.5, p=0.29$ ). For males, piloerection frequency changed across the study (Friedman ANOVA: $\chi^{2}=8.166, d f=2, p=0.016$ ), and a significant decrease was observed only during P2 in relation to $\mathrm{BP}$ and $\mathrm{P} 1$ (Wilcoxon: $\mathrm{BP} * \mathrm{P} 1 ; z=-1.67$, $p=0.09 ; \quad \mathrm{BP} * \mathrm{P} 2 ; \quad z=-2.11, \quad p=0.034 ; \quad \mathrm{P} 1 * \mathrm{P} 2 ;$ $z=-2.89, p=0.003$ ), but not for BP versus P1 (Wilcoxon: BP*P1; $z=-1.67, p=0.09$ ) (Fig. 2b). Females also changed piloerection behavior between the phases (Friedman ANOVA: $\chi^{2}=14.98, d f=2, p=0.001$ ) and a significant decrease was also observed in $\mathrm{P} 2$ compared to $\mathrm{BP}$ and P1 (Wilcoxon: $\mathrm{BP} * \mathrm{P} 2 ; z=-3.229, p=0.001$; $\mathrm{P} 1 * \mathrm{P} 2 ; z=-3.404, p=0.001)$ but not for $\mathrm{BP}$ versus $\mathrm{P} 1$ (Wilcoxon: BP*P1; $z=-0.831, p=0.406$ ) (Fig. 2b).

Autogrooming No sex differences were detected in total autogrooming behavior (Mann-Whitney, $U=5864.5$, $p=0.16$ ). The duration of male autogrooming changed between the phases (Friedman ANOVA: $\chi^{2}=16.53$, $d f=2, p=0.001)$, showing a significant reduction in $\mathrm{P} 1$ when compared to BP and P2 (Wilcoxon: BP*P1; $z=-3.000, p=0.002 ; \mathrm{P} 1 * \mathrm{P} 2 ; z=-2.723, p=0.006)$, but not between $\mathrm{BP}$ and $\mathrm{P} 2$ (Wilcoxon: BP*P2; $z=-0.125$, $p=0.900$ ) (Fig. 2c). Females did not display a significant interphase variation in autogrooming behavior (Friedman ANOVA: $\chi^{2}=0.706, d f=2, p=0.703$ ) (Fig. $2 \mathrm{c}$ ). 
Fig. 1 a Mean $( \pm$ SE) fecal cortisol levels (ng/g) for male and female Callithrix jacchus during each phase 1 ( $B P$ baseline phase, $P 1$ moving to same-size cages, $P 2$ moving to smaller-size cages) (males: P1*BP: $p=0.9$; $\mathrm{P} 2 * \mathrm{BP}$ : $p=0.02 ; \mathrm{P} 2 * \mathrm{P} 1 ; p=0.02$; females: P1*BP: $p=0.001$; $\mathrm{P} 2 * \mathrm{BP}: p=0.07 ; \mathrm{P} 2 * \mathrm{P} 1$ : $p=0.15)$. b Daily mean $( \pm \mathrm{SE})$ of fecal cortisol levels during experiment $\mathrm{I}$ in males and females. GLM test, Fisher's post-hoc, $p<0.05$. The symbol asterisk indicates a significant statistical difference and ash a statistical tendency to different cortisol levels between the respective study phase and those represented by the number beside the symbol. Letters $f$ and $m$ indicate females and males, respectively
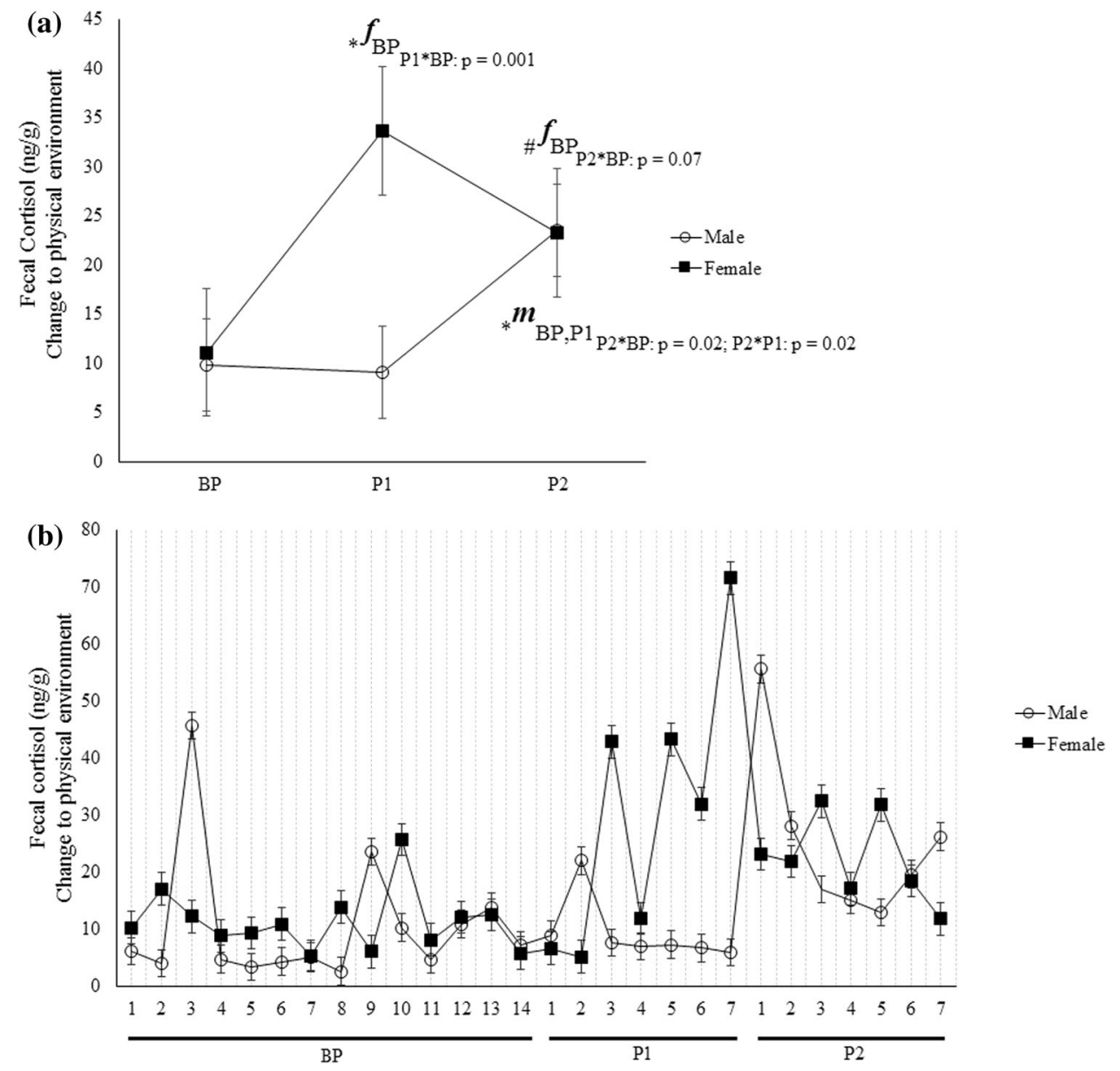

\section{Statistical correlation}

Males exhibited a significant negative correlation between piloerection and autogrooming in phases P1 and P2 (Spearman's correlation, $p=0.001, r \mathrm{~s}=-0.4$ ) (Table 1). For males, no correlations between cortisol and autogrooming (Spearman's correlation, $p=0.124, r s=0.18$ ), cortisol and scent marking (Spearman's correlation, $p=0.08, r s=-0.2$ ), or cortisol and piloerection (Spearman's correlation, $p=0.366, r s=-0.1$ ) were found in P1 and P2. Moreover, there was no correlation between scent making and autogrooming (Spearman's correlation, $p=0.22, r s=-0.13$ ) or scent making and piloerection (Spearman's correlation, $p=0.08, r s=0.2$ ) in P1 and P2.

For females, a significant positive correlation between piloerection and scent marking (Spearman's correlation, $p<0.05, r s=0.0001$ ) and a negative correlation between piloerection and autogrooming were observed during P1 and P2 (Spearman's correlation, $p=0.01, r s=-0.3$ ) (Table 1). However, no correlations between cortisol and autogrooming (Spearman's correlation, $p=0.758$, $r s=-0.04$ ), cortisol and scent marking (Spearman's correlation, $p=0.835, r s=0.02$ ), or cortisol and piloerection (Spearman's correlation, $p=0.649, r s=0.06$ ) were observed in P1 and P2. In addition, no correlation was found between scent making and autogrooming (Spearman's correlation, $p=0.78, r s=-0.03$ ) in $\mathrm{P} 1$ and $\mathrm{P} 2$.

\section{Experiment II: first-time pair formation}

\section{Cortisol}

Females showed higher total mean cortisol levels $(\mu=14.25 \pm 1.91 \mathrm{ng} / \mathrm{g})$ than males $(\mu=7.9 \pm 1.97 \mathrm{ng} / \mathrm{g})$ (GLM; sex: $\left.F=5.31, d f=1, p=0.02, \eta_{p}^{2}=0.058\right)$. Both males and females showed a statistically significant increase in cortisol levels after pairing (P3) (GLM; phases: $F=6.99$, $d f=1, p=0.009, \eta_{p}^{2}=0.075$ ) (Fig. 3a). A qualitative analysis of the progression of cortisol response revealed that males recovered their cortisol levels the day after pair formation (P3), exhibiting a random variation pattern, with data not fitting any curve distribution $(\beta=0.168$, but curve estimation tests were all non-significant). On the other hand, females exhibited a more intense and longer-lasting cortisol 

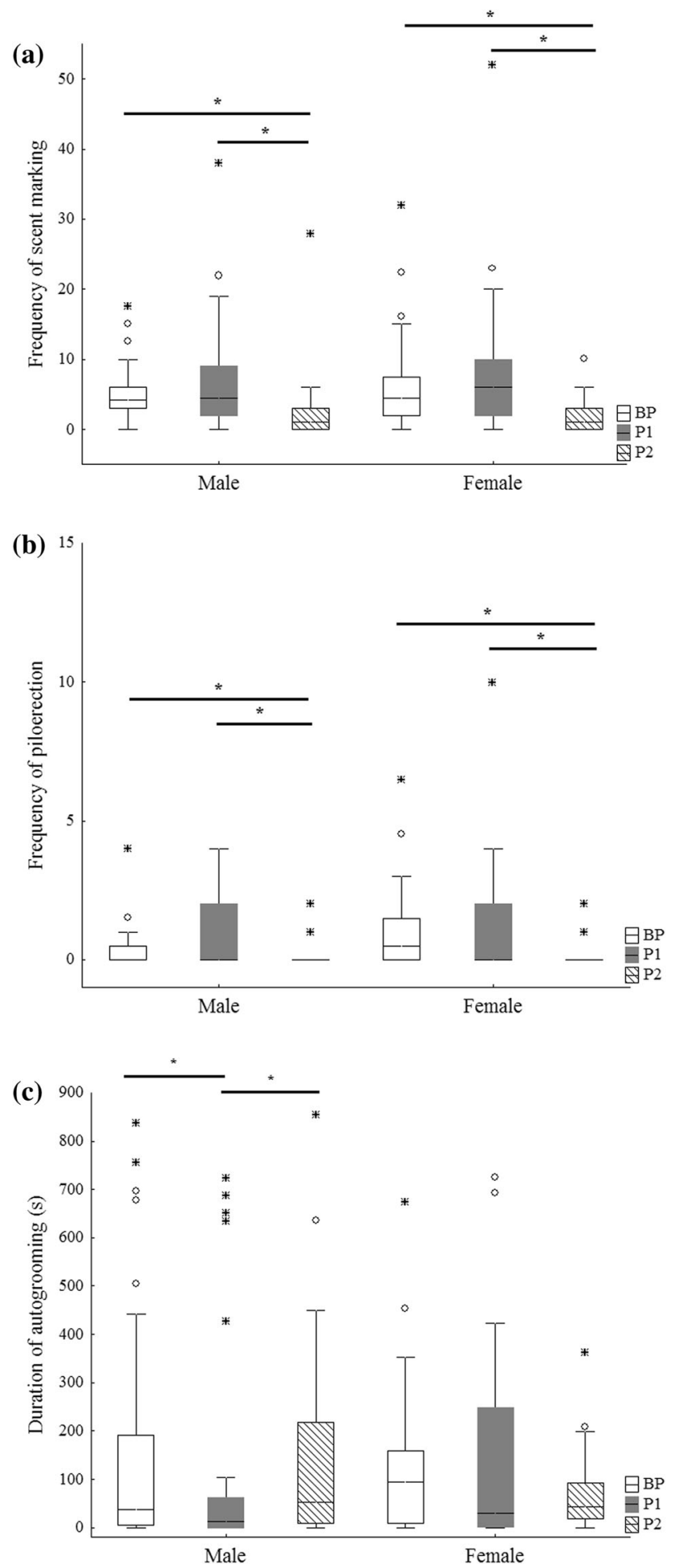

Fig. 2 Median (quartiles 25 and 75) behavior frequencies and duration of male and female Callithrix jacchus activities in experiment I ( $B P$ baseline phase, $P 1$ moving to same-size cages, $P 2$ moving to smaller cages). Frequencies of: a scent marking, b piloerection and c duration of autogrooming in seconds for both males and females. Friedman ANOVA test was performed according to sex and the Wilcoxon test was used to compare interphase differences $(p<0.05)$. The symbol asterisk indicates a significant difference in behaviors between the respective study phase and those represented by the number beside the symbol
Table 1 Correlations between behaviors along of stress phases

\begin{tabular}{lll}
\hline Sex & P1 + P2 & P3 \\
\hline Male & Pilo * AutoG $(r \mathrm{~s}=-0.4)$ & Pilo * ScentM $(r \mathrm{~s}=0.5)$ \\
Female & Pilo * ScentM $(r \mathrm{~s}=0.56)$ & Pilo * ScentM $(r \mathrm{~s}=0.49)$ \\
& Pilo * AutoG $(r \mathrm{~s}=-0.3)$ & Pilo * AutoG $(r \mathrm{~s}=-0.38)$ \\
\hline $\begin{array}{l}\text { Spearman's correlation test, } p<0.05 \\
\text { Pilo individual piloerection, ScentM } \\
\text { autogrooming }\end{array}$ & scent marking, AutoG
\end{tabular}

increase that fit to a cubic distribution and was detected up to 5 days after paring $(F=19.114, p=0.019, \beta=2.518)$ (Fig. 3b).

\section{Behaviors}

No intersex differences were observed in scent marking, (Mann-Whitney: $U=1483.6, \quad p=0.263$ ) and autogrooming (Mann-Whitney: $\quad U=1395.5, \quad p=0.394$ ) behaviors. However, females showed higher piloerection frequencies than males (Mann-Whitney: $U=1226.5$, $p=0.043$ ).

Only females showed a variation in behaviors across the phases and increased piloerection frequency before the pair formation phase (Wilcoxon: $z=2.941, d f=1, p=0.03$ ) (Fig. 4a). For all other behaviors, males (piloerection, Wilcoxon: $z=0.11, d f=1, p=0.909$; scent-marking, Wilcoxon: $z=1.65, d f=1, p=0.09$; autogrooming, Wilcoxon: $z=0.588, d f=1, p=0.556$ ) and females showed no change during P3 (after pairing) (scent-marking, Wilcoxon: $z=0.769, d f=1, \quad p=0.441$; autogrooming, Wilcoxon: $z=0.672, p=0.501$ ) (Fig. 4).

\section{Statistical correlations}

For males and females after pairing (P3), scent marking and individual piloerection were positively correlated (Spearman's correlation, males: $p=0.0001, r \mathrm{~s}=0.50$; females: $p=0.01, r \mathrm{~s}=0.49$ ). Individual piloerection in females was negatively correlated with autogrooming $(p=0.04, r \mathrm{~s}=-0.38)$ (Table 1). No correlations between cortisol and autogrooming (Spearman's correlation, males: $p=0.3, r \mathrm{~s}=0.25$; females: $=0.4, r \mathrm{~s}=-0.19)$, cortisol and scent marking (Spearman's correlation, males: $p=0.656, r \mathrm{~s}=0.11$; females: $p=0.394, r \mathrm{~s}=0.2$ ), or cortisol and piloerection (Spearman's correlation, males: $p=0.858, \quad r s=0.04$; females: $p=0.561, r s=0.13$ ) were observed for males and females in P3. Furthermore, no correlations were found between autogrooming and scent marking (Spearman's correlation, $p=0.08$, $r \mathrm{~s}=0.33$ ) or autogrooming and piloerection (Spearman's correlation, $p=0.5, r \mathrm{~s}=0.37$ ) in males, and no correlation was observed between autogrooming and scent 
Fig. 3 a Mean $( \pm$ SE) fecal cortisol levels $(\mathrm{ng} / \mathrm{g})$ of male and female Callithrix jacchus before and after the pair formation experiment (BP2 = baseline phase 2; P3 = pair formation phase). b Daily mean $( \pm$ SE) fecal cortisol levels during experiment II in males and females. GLM and Fisher's post-hoc tests, $p<0.05$. The symbol asterisk indicates a statistically significant difference in cortisol levels between the respective study phase and those represented by the number beside the symbol
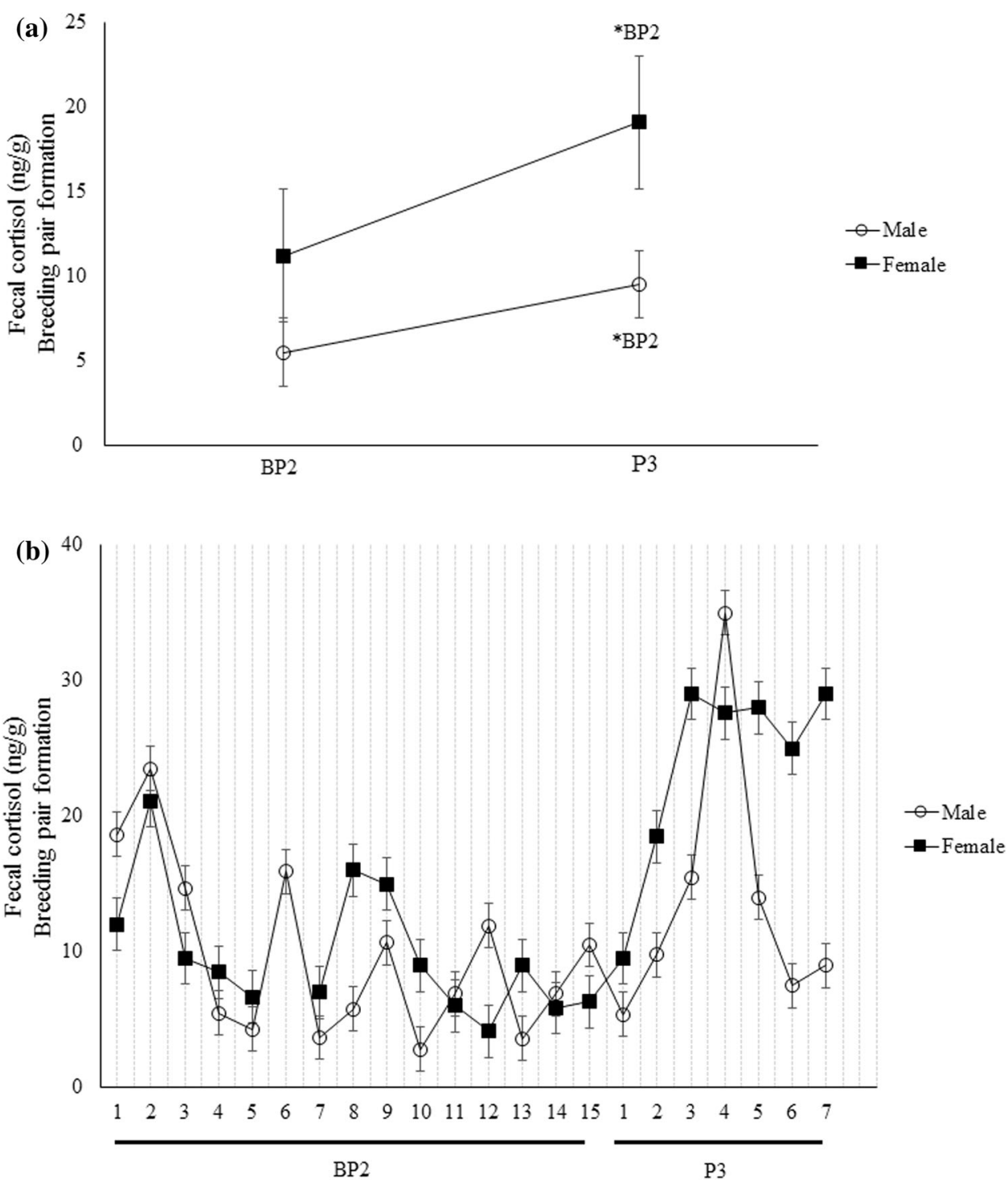

marking (Spearman's correlation, $p=0.181, r \mathrm{~s}=-0.26$ ) in females.

\section{Discussion}

To comply with maintenance and research protocols, general management, introduction of enrichment programs, and implementation of reproductive monitoring in colonies, animals are routinely moved to different cages, where pairs are formed. The current study investigated the stress responses of common marmosets to these procedures.

As expected, females exhibited higher mean total cortisol levels in feces than males, as previously demonstrated by Sousa et al. (2002), and a sexually dimorphic cortisol pattern was observed in response to the challenges of moving the animals to same-size or smaller cages and of first-time pair formation. When animals were moved individually to a similar-size cage (P1) than that of the baseline phase, only females showed an increase in cortisol levels. When animals were placed in smaller cages (P2), males exhibited a significant increase in cortisol, whereas females showed a trend toward an increase in this hormone. Thus, these data indicate that mechanisms of HPA regulation differ between sexes. It is noteworthy that when female or male isossexual dyads of adult $C$. jacchus were moved together to a cage with similar spatial dimensions, like those of Phase 1, no change in cortisol was recorded (Galvão-Coelho et al. 2012). This indicates that social support, mainly to the female $C$. jacchus, may buffer major stress reactions to a novelty change, as occurred when they were moved to new cages in this study. Another study using $C$. jacchus observed a behavioral response to stress, with an increase in piloerection, startle response (flight 

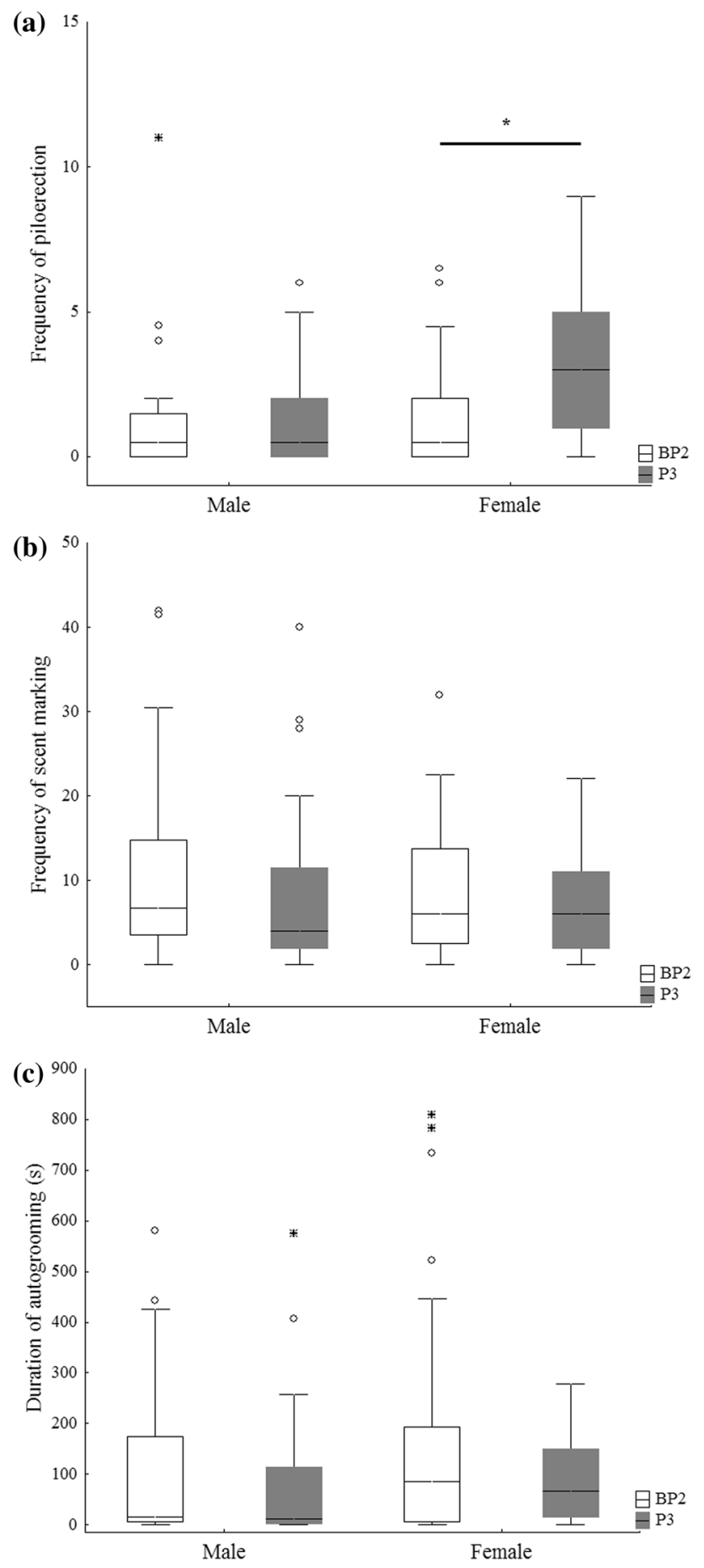

Fig. 4 Median (quartiles 25 and 75) behavior frequency and duration of male and female Callithrix jacchus activities in experiment II (BP2 baseline phase 2, $P 3$ pair formation phase). a Piloerection frequency, b scent marking frequency and c autogrooming duration in seconds for both sexes. The Wilcoxon test was used to analyze interphase differences for each sex $(p<0.05)$. The symbolasterisk indicates a significant difference in behaviors between the respective study phase and those represented by the number beside the symbol reaction), aggression, and stereotype behaviors when a heterosexual dyad was moved to a smaller cage $(60 \times 60$ $\times 82 \mathrm{~cm}$ ) (Kitchen and Martin 1996). The authors argue that increasing the undesirable contact that occurs in small cages might be responsible for the stress response observed. Therefore, dyadic arrangements are recommended if it is necessary to move animals to another similar size cage during routine management. However, social support does not seem to be beneficial in situations of spatial restriction.

Additionally, qualitative statistical analysis showed a marked increase and rapid cortisol recovery to basal levels in males, after being moved to a smaller cage (P2), while females exhibited a more variable and longer-lasting cortisol response subsequent to both cage changes. This type of response likely occurs in females since they develop adaptation mechanisms to deal with the constraints caused by emigration behaviors that are more frequent in this sex. Thus, when they are living in the natural environment, they emigrate from the natal group in order to seek vacancies to fill and integrate new social groups in an attempt at obtaining reproductive success (Sousa et al 2009; Yamamoto et al. 2010). A similar dimorphic sexual response to moving was observed when male and female $C$. kuhli were isolated in small cages (Smith and French 1997). However, in this case the animals were monitored only on the day after moving, allowing only a limited comparison. Nevertheless, this finding reinforced the biological significance of this difference as being a reflex of physiological adaptations to cope with natural challenges. In common marmosets, internal fertilization, lactation, and the transition from weaning offspring to solid food diet (Ximenes and Sousa 1996) require the participation of females to ensure infant survival. Thus, females must adapt to deal with these challenges differently from males since, in addition to direct reproductive costs, they are more frequently exposed to forced and prolonged isolation.

In regard to behavioral responses, males and females exhibited similar reactions to being moved to a new cage, except for autogrooming. During P1, both males and females showed no change in scent marking frequency and piloerection behaviors, but while females maintained their autogrooming at similar to baseline levels, males exhibited a decrease in the duration of this behavior when compared to BP. This sexually dimorphic response to changing cages (P1) may also be associated with differences in the regulation of stress responses in females, when compared to male coping strategies, since females tend to emigrate more frequently in the wild (Sousa et al. 2009). Therefore, they could exhibit behavioral adaptations, such as the use of autogrooming, to buffer sustained stress caused by 
environmental changes. Autogrooming is considered a tension-reducing behavior since it induces oxytocin secretion and decreases the activation of stress response systems (DeVries et al. 2007; Wittig et al. 2008). For males, the HPA regulation mechanism seems not to involve this behavior to cope with this specific context. However, as shown by Taylor et al. (2015) in Callithrix geoffroyi, males and females used grooming as a buffer to cope with acute social isolation.

During P2, female autogrooming remained constant in relation to other phases, while that of males returned to BP levels, increasing its duration compared to P1. Corroborating the calming effect of autogrooming, we found a significant inverse correlation between this behavior and individual piloerection for both males and females in stress phases P1 and P2 of experiment I. Both males and females decreased their scent marking and piloerection behaviors in P2 compared to baseline and P1. Moreover, males and females showed a positive correlation between piloerection and scent marking in P1 and P2. Scent marking and piloerection are territorial behaviors that tend to increase in free ranging animals exposed to new environments (Rylands 1985). In terms of captive studies, these behaviors are associated with anxiety and stress exposure (Barros and Tomaz 2002; Dettling et al. 2002). Furthermore, piloerection is considered an indicator of sympathetic autonomic nervous system activation (Dettling et al. 2002).

The overall decrease in scent marking and piloerection in both males and females during P2 may be due to the reduced size of the new cage (Kitchen and Martin 1996). The decline in these behaviors, associated with increased cortisol, is similar to that found in chronic stress situations where animals show similar profiles to those of depression (Hennessy et al. 2005). An isolated social condition in restricted spatial environments generally inhibits the development of normal coping strategies and induces the development of bizarre behavior patterns, or even the absence of their expression. However, to confirm that the behavioral and physiological findings demonstrated in the current study actually express a depression-like condition, other indicators such as locomotor activity levels (Shively et al. 2005), anhedonia (Shively and Willard 2012), and body weight (Shively and Willard 2012) must be investigated.

The fact that adult common marmoset males and females exhibited differences in both endocrine and behavioral responses when moved to different cages (experiment I) supports the second hypothesis of this study. However, the first supposition was only valid for males that showed a more intense cortisol response to spatial restriction and not for females, who were more reactive to novelty.

The results of experiment II showed that both males and females demonstrated the same hormonal response in the acute pair formation condition, characterized by increased cortisol levels. Once again, qualitative statistical analysis indicated that males display a cortisol response shortly after pair formation, while females exhibit a more variable and longer-lasting cortisol response. This response is also similar to that found for common marmoset females moved from their natal group and first paired with unfamiliar males (Ziegler and Sousa 2002). According to this study, which measured hormones only in females, the increase in cortisol was significantly higher in the first 20 days following transfer to the paired condition when compared to the last 20 days in the natal group. Additionally, the cortisol increase when females returned to their natal group suggests psychosocial stress due to social change and the introduction of an unfamiliar male. In the first 10 weeks after first-time unfamiliar pair formation, during a longitudinal behavioral study, Silva and Sousa (1997) found that social interaction between males and females was minimal at the beginning of pairing, showing significant changes only after the third week of cohabitation. Thus, after pair formation, a minimum 3-week adaptation period seems to be necessary before the start of any experimental procedure.

Females also demonstrated an increase in individual piloerection frequency during the pair formation phase, indicating activation of the sympathetic autonomic nervous system (Dettling et al. 2002). Males and females showed a significant positive correlation across piloerection and scent marking in P3, while only females displayed a significant inverse correlation between piloerection and autogrooming. These correlations corroborate previous suggestions that scent marking and piloerection are stress markers and that autogrooming acts as tension-reducing mechanism. Thus, in addition to HPA, the sympathetic autonomic component of stress response was expressed by cortisol and behavioral analysis in marmosets, reinforcing the importance of studying both hormones and behavior to better characterize basal conditions in captivity. The behavioral and hormonal results found in experiment II support the third hypothesis of an acute sexual dimorphic response for cortisol and behaviors, where males recovered cortisol levels faster (on the day after pairing) than females, who maintained increased cortisol levels during the first 5 days.

The "Main Effect Theory" suggests that distress levels decline in the presence of a social support system (Cohen and Wills 1985), one of the benefits of pair formation in captivity. Studies of non-human primates demonstrate the benefits of group life and social support during crisis situations, mitigating the harmful effects of stress (GalvãoCoelho et al. 2012). Notwithstanding these benefits, behavioral and hormonal components of stress systems are activated during pair formation in both male and female $C$. 
jacchus, and a comparable increase in cortisol levels after breeding pair formation has been demonstrated in common marmosets (Leão 2001). In Callithrix geoffroyi, males and females, isolated or living in family groups, exhibited increased cortisol levels when paired with a sexual partner. Therefore, previous research and this study suggest that the efficacy of social support during the stress response is not a generalized event, but rather depends on certain factors such as species, gender, pairing duration, partner familiarity, and previous living condition (Gerber et al. 2002; Galvão-Coelho et al. 2012). Thus, pair-formation demands an extended temporal period to attain stability and achieve baseline levels of physiological and behavioral functioning.

The International Primatological Society, in accordance with international guidelines for the suitable care and breeding of non-human primates, recommends that the minimum cage height be $1.5 \mathrm{~m}$ (Buchanan-Smith et al. 2007). It is also recommend that the animals remain in social groups, avoiding social isolation. In Brazil, animal care legislation (IN 169) stipulates that common marmosets be housed in family groups in $5 \mathrm{~m}^{3}$ cages. However, neither guideline provides recommendations regarding the routine procedures that regularly demand isolating animals (e.g., due to sickness or disease) in cages with restricted spatial dimensions. The results of the current study show that captivity management introduces endocrine and behavioral alterations that can last for more than $24 \mathrm{~h}$, especially in females. Changes in the baseline levels of behavioral and endocrine variables must be accounted for when analyzing and discussing experimental findings. Although cortisol levels rise and the changes in behavioral patterns detected in this New World cooperative breeding primate may potentially be extended to other primates, the specific traits of each species, such as sex, age, pairing duration, partner familiarity, previous living condition, and type of captivity (zoos or biomedical centers) must be considered. For instance, Cynomolgus monkeys (Macaca fascicularis), unlike common marmosets, do not exhibit sexual dimorphism in their response to new environments and spatial restriction, and are more reactive to new environments than to spatial restriction (Crockett et al. 1995). Indeed, singlehoused female Cynomolgus monkeys are more behaviorally reactive to small transport cages than pair-housed females (Schapiro et al. 2012). For common marmosets, a number of studies have shown that this species exhibits different endocrine and behavioral responses depending on the nature of the stressor, sex, baseline cortisol, and age (GalvãoCoelho et al. 2008, 2012, Sousa et al. 2015).

Finally, we suggest that stress during routine management might be minimized and interspersed in sufficient time to allow endocrine and behavioral recovery, to avoid chronic activation of the stress response system, which may compromise the welfare of the animals and trigger physical and mental pathologies (McEwen 2000). We recommend more studies on the impacts of routine colony management so that protocols can be devised and included in official husbandry guidelines in order to improve welfare conditions during the maintenance of non-human primates in captivity, and reduce bias that can skew study results.

Acknowledgments We would like to thank Edinolia Câmara, Antonio B. da Silva, and Geniberto C. dos Santos for animal care, and José Flávio V. Coutinho and Janaína Nitta for veterinary care.

\section{Compliance with ethical standards}

Conflict of interest The authors declare they have no conflicts of interest.

\section{References}

Altmann J (1974) Observational study of behavior: sampling methods. Behaviour 49:227-267

Baker KC (2007) Enrichment and primate centers: closing the gap between research and practice. J Appl Anim Welf Sci 10:49-54

Baker KC, Weed JL, Crockett CM, Bloomsmith MA (2007) Survey of environmental enhancement programs for laboratory primates. Am J Primatol 69:377-394

Balcombe JP, Barnard ND, Sandusky C (2004) Laboratory routines cause animal stress. Contemp Top Lab Anim Sci 43:42-51

Barros M, Tomaz C (2002) Non-human primate models for investigating fear and anxiety. Neurosci Biobehav Rev 26:187-201

Boere V (2001) Behavior and environment enrichment. In: Fowler ME, Cubas ZS (eds) Biology, medicine and surgery of South American wild animals. Iowa University Press, Ames, pp 263-266

Buchanan-Smith H, Jones-Engel L, Farmer K, Prescott M, FitchSnyder H, Tayor S (2007) IPS international guidelines for the acquisition, care and breeding of nonhuman primates. International Primatological Society

Charmandari E, Tsigos C, Chrousos G (2005) Endocrinology of the stress response. Annu Rev Physiol 67:259-284

Cohen S, Wills TA (1985) Stress, social support, and the buffering hypothesis. Psychol Bull 98:310-357

Crockett CM, Bowers CL, Shimoji M, Leu M, Bowden DM, Sackett GP (1995) Behavioral responses of longtailed macaques to different cage sizes and common laboratory experiences. J Comp Psychol 109:368-383

Dawkins MS (2004) Using behaviour to assess animal welfare. Anim Welf 13:19-30

Dettling A, Feldon J, Pryce CR (2002) Early deprivation and behavioral and physiological responses to social separation novelty in the marmoset. Pharmacol Biochem Behav 73:259-269

DeVries A, Craft TKS, Glasper ER, Neigh GN, Alexander JK (2007) Social influences on stress responses and health. Psychoneuroendocrinology 32:587-603

Dixson AF, Lunn SF (1987) Post-partum changes in hormones and sexual behaviour in captive groups of marmosets (Callithrix jacchus). Physiol Behav 41:577-583

Erkert HG (1989) Characteristics of the circadian activity rhythm in common marmosets (Callithrix jacchus). Am J Primatol 17:271-286

Galvão-Coelho NL, Silva HPA, Leão AC, Sousa MBC (2008) Common marmosets (Callithrix jacchus) as a potential animal 
model for studying psychological disorders associated with high and low responsiveness of hypothalamic-pituitary-adrenal axis. Rev Neurosci 19:187-201

Galvão-Coelho NL, Silva HP, de Sousa MB (2012) The influence of sex and relatedness on stress response in common marmosets (Callithrix jacchus). Am J Primatol 74:819-827

Gerber P, Schnell CR, Anzenberger G (2002) Behavioral and cardiophysiological responses of common marmosets (Callithrix jacchus) to social and environmental changes. Primates 43:201-216

Hennessy MB, Mendoza SP, Kaplan JN (2005) Behavior and plasma cortisol following brief peer separation in juvenile squirrel monkeys. Am J Primatol 3:143-151

Kishi N, Sato K, Sasaki E, Okano H (2014) Common marmoset as a new model animal for neuroscience research and genome editing technology. Dev Growth Differ 56:53-62

Kitchen AM, Martin AA (1996) The effects of cage size and complexity on the behaviour of captive common marmosets, Callithrix jacchus. Lab Ani 30:317-326

Kohu K, Yamabe E, Matsuzawa A, Onda D, Suemizu H, Sasaki E, Tanioka Y, Yagita H, Suzuki D, Kametani Y, Takai T, Toyoda A, Habu S, Satake M (2008) Comparison of 30 immunity-related genes from the common marmoset with orthologues from human and muse. Tohoku J Exp Med 215:167-180

Lacreuse A, Chang J, Metevier CM, Laclair M, Meyer JS, Ferris CM (2014) Oestradiol modulation of cognition in adult female marmosets (Callithrix jacchus). J Neuroendocr 26:296-309

Leão, AC (2001) Variação hormonal e comportamental em sagui comum (Callithrix jacchus), durante as fases de formação, separação e reunião do par heterosexual. M.Sc. Dissertation, Universidade Federal do Rio Grande do Norte, Brazil (in Portuguese)

Mansfield K (2003) Marmoset models commonly used in biomedical research. Comp Med 53:383-392

McEwen B (2000) The neurobiology of stress: from serendipity to clinical relevance. Brain Res 886:172-189

Newberry RC (1993) The space-time continuum and its relevance to farm animals. Etologia 3:219-234

Phillips KA, Hambright MK, Hewes K, Schilder BM, Ross CN, Tardif SD (2015) Take the monkey and run. J Neurosci Methods 248:27-31

Prasad S, Humphreys I, Kireta S, Gilchrist RB, Bardy P, Russ GR, Coates PTH (2007) The common marmoset as a novel preclinical transplant model: identification of new MHC class II DRB alleles and prediction of in vitro alloreactivity. Tissue Antigens 69:72-75

Raminelli JLF, Sousa MBC, Cunha MS, Barbosa MFV (2001) Morning and afternoon patterns of fecal excretion of cortisol among reproductive and non-reproductive male and female common marmosets, Callithrix jacchus. Biol Rhythm Res 32:159-167

Ruby S, Buchanan-Smith HM (2015) The effects of individual cubicle research on the social interactions and individual behavior of brown capuchin monkeys (Sapajus apella). Am J Primatol 77:1097-1108

Rylands AB (1985) Tree-gouging and scent-marking by marmosets. Anim Behav 33:1365-1367

Schapiro SJ, Lambeth SP, Jacobsen KR, Williams LE, Nehete BN, Nehete PN (2012) Physiological and welfare consequences of transport, relocation, and acclimatization of chimpanzees (Pan troglodytes). Appl Anim Behav Sci 137:183-193
Shively CA, Willard SL (2012) Behavioral and neurobiological characteristics of social stress versus depression in nonhuman primates. Exp Neurol 233:87-94

Shively CA, Register TC, Friedman DP, Morgan TM, Thompson J, Lanier T (2005) Social stress-associated depression in adult female cynomolgus monkeys (Macaca fascicularis). Biol Psychol 69:67-84

Silva HP, Sousa MBC (1997) The pair-bond formation and its role in the stimulation of reproductive function in female common marmosets, Callithrix jacchus. Int J Primatol 18:387-400

Smith TE, French JA (1997) Psychosocial stress and urinary cortisol excretion in marmoset monkeys (Callithrix kuhli). Physiol Behav. 62:225-232

Sousa MBC, Ziegler T (1998) Diurnal variation on the excretion patterns of steroids in common marmoset (Callithrix jacchus) females. Am J Primatol 46:105-117

Sousa MBC, Silva HPA, Leão AC (2002) Sexual differences on behavior and fecal cortisol using the separation paradigm in common marmosets, Callithrix jacchus. In: Abstracts, IPS conference, Beijing, p 94

Sousa MBC, Albuquerque ACSR, Yamamoto ME, Araújo A, Arruda MF (2009) Emigration as a reproductive strategy of the common marmoset (Callithrix jacchus). In: Ford SM Porter LM Davis LC (eds) The smallest anthropoids: the marmoset/callimico radiation. Springer Press, New York, pp 167-182

Sousa MBC, Galvão ACM, Sales CJR, Galvão-Coelho NL (2015) Endocrine and cognitive adaptations to cope with stress in immature common marmosets (Callithrix jacchus): sex and age matter. Front Psychiatry. doi:10.3389/fpsyt.2015.00160

Stevenson MF, Poole TB (1976) An ethogram of common marmoset (Callithrix jacchus): general behavioral repertoire. Anim Behav. doi:10.1016/S0003-3472(76)80053-X

Stevenson MF, Rylands AB (1988) The marmosets, genus Callithrix. In: Mittermeier RA, Rylands AB, Coimbra-Filho A, Fonseca $\mathrm{GAB}$ (eds) Ecology and behavior of neotropical primates. Contagem, Littera Maciel, pp 131-222

't Hart BA, Abbott DH, Nakamura K, Fuchs E (2012) The marmoset monkeys: a multi-purpose preclinical and translational model of human biology and disease. Drug Discov Today 17:1160-1165

Taylor JH, Mustoe AC, Hochfelder B, French JA (2015) Reunion behavior after social separation is associated with enhanced HPA recovery in young marmoset monkeys. Psychoneuroendocrinology 57:93-101

Wittig RM, Crockford C, Lehmann J, Whitten PL, Seyfarth RM, Cheney DL (2008) Focused grooming networks and stress alleviation in wild female baboons. Horm Behav 54:170-177

Ximenes MFFM, Sousa MBC (1996) Family composition and the characteristics of parental care during the nursing phase of captive common marmosets (Callithrix jacchus). Primates 37:175-183

Yamamoto ME, Araújo A, de Sousa MBC, de Fátima Arruda M (2010) Social organization in Callithrix jacchus: cooperation and competition. Adv Study Behav 42:259-273

Young RJ (2003) Environmental enrichment for captive animals. Blackwell Science, Oxford

Ziegler TE, Sousa MBC (2002) Parent-daughter relationships and social controls on fertility in female common marmosets, Callithrix jacchus. Horm Behav 42:356-367 